Washington University School of Medicine Digital Commons@Becker

\title{
Cost-effectiveness of peer-delivered interventions for cocaine and alcohol abuse among women: A randomized controlled trial
}

Jennifer Prah Ruger

Yale University School of Medicine

Arbi Ben Abdallah

Washington University School of Medicine in St. Louis

Craig Luekens

Yale University School of Medicine

Linda Cottler

Washington University School of Medicine in St. Louis

Follow this and additional works at: https://digitalcommons.wustl.edu/open_access_pubs

Part of the Medicine and Health Sciences Commons

Please let us know how this document benefits you.

\section{Recommended Citation}

Prah Ruger, Jennifer; Ben Abdallah, Arbi; Luekens, Craig; and Cottler, Linda, "Cost-effectiveness of peerdelivered interventions for cocaine and alcohol abuse among women: A randomized controlled trial." PLoS ONE. 7, 3. 1-12. (2012).

https://digitalcommons.wustl.edu/open_access_pubs/1012

This Open Access Publication is brought to you for free and open access by Digital Commons@Becker. It has been accepted for inclusion in Open Access Publications by an authorized administrator of Digital Commons@Becker. For more information, please contact vanam@wustl.edu. 


\title{
Cost-Effectiveness of Peer-Delivered Interventions for Cocaine and Alcohol Abuse among Women: A Randomized Controlled Trial
}

\author{
Jennifer Prah Ruger ${ }^{1 *}$, Arbi Ben Abdallah ${ }^{2}$, Craig Luekens ${ }^{1}$, Linda Cottler ${ }^{2}$ \\ 1 Department of Public Health, Yale University School of Medicine, New Haven, Connecticut, United States of America, 2 Washington University School of Medicine, St. \\ Louis, Missouri, United States of America
}

\begin{abstract}
Aims: To determine whether the additional interventions to standard care are cost-effective in addressing cocaine and alcohol abuse at 4 months (4 M) and 12 months (12 M) from baseline.

Method: We conducted a cost-effectiveness analysis of a randomized controlled trial with three arms: (1) NIDA's Standard intervention (SI); (2) SI plus a Well Woman Exam (WWE); and, (3) SI, WWE, plus four Educational Sessions (4ES).

Results: To obtain an additional cocaine abstainer, WWE compared to SI cost $\$ 7,223$ at $4 \mathrm{M}$ and $\$ 3,611$ at $12 \mathrm{M}$. Per additional alcohol abstainer, WWE compared to SI cost $\$ 3,611$ and $\$ 7,223$ at $4 \mathrm{M}$ and $12 \mathrm{M}$, respectively. At $12 \mathrm{M}$, 4ES was dominated (more costly and less effective) by WWE for abstinence outcomes.

Conclusions: To our knowledge, this is the first cost-effectiveness analysis simultaneously examining cocaine and alcohol abuse in women. Depending on primary outcomes sought and priorities of policy makers, peer-delivered interventions can be a cost-effective way to address the needs of this growing, underserved population.
\end{abstract}

Trial Registration: ClinicalTrials.gov NCT01235091

Citation: Prah Ruger J, Abdallah AB, Luekens C, Cottler L (2012) Cost-Effectiveness of Peer-Delivered Interventions for Cocaine and Alcohol Abuse among Women: A Randomized Controlled Trial. PLoS ONE 7(3): e33594. doi:10.1371/journal.pone.0033594

Editor: Jeremy Miles, Research and Development Corporation, United States of America

Received November 18, 2011; Accepted February 13, 2012; Published March 20, 2012

Copyright: (c) 2012 Prah Ruger et al. This is an open-access article distributed under the terms of the Creative Commons Attribution License, which permits unrestricted use, distribution, and reproduction in any medium, provided the original author and source are credited.

Funding: This study was supported by the National Institutes of Health (NIH) National Institute on Drug Abuse ([NIDA] grant R01DA11622). JR was supported by a Career Development Award from the NIH (NIDA grant K01DA01635810). The funders had no role in study design, data collection and analysis, decision to publish, or preparation of the manuscript.

Competing Interests: The authors have declared that no competing interests exist.

*E-mail: jennifer.ruger@yale.edu

\section{Introduction}

Over $9 \%$ of the total US population (23.1 million aged 12 or older) was in need of treatment for an illicit drug or alcohol use problem in 2008 [1]. Among females, the percentage of illicit drug users rose from $5.8 \%$ in 2007 to $6.8 \%$ in 2010 ; the number of users increased from $10.4 \%$ to $11.2 \%$ over the same period among males [1,2]. Alcohol and illicit drug use were the third and ninth leading causes of preventable death in 2000, associated with 85,000 and 17,000 deaths, respectively [3]. Moreover, the economic costs of drug abuse in the US have increased $5.3 \%$ annually from 1992 to 2002 , reaching a total of $\$ 180.9$ billion, $\$ 16$ billion of which were health-related [4]. Despite this rise in the costs of substance abuse, health care spending on substance abuse only rose $4.1 \%$ annually, whereas total health care spending rose by $6.5 \%$.

A number of behavioral interventions have shown effectiveness in treating cocaine [5,6] and alcohol abuse [7]. The Women Teaching Women intervention (WTW) was designed to reduce substance use and high-risk sexual behaviors through a holistic, peer-delivered intervention [8]. A randomized, controlled trial (RCT) examined three arms of WTW: (1) a modified National
Institute on Drug Abuse (NIDA) Cooperative Agreement Standard Intervention (SI); (2) SI and a field-based Well Woman Exam (WWE); and (3) SI, WWE, and four Educational Sessions (4ES). The RCT took place in St. Louis, where, aside from alcohol, cocaine caused the greatest amount of treatment admissions in 2007 and remains a major drug problem $[9,10]$. This study examined ten outcomes related to cocaine and alcohol use; a companion study analyzed the cost-effectiveness of these interventions for preventing, STDs and HIV [11]. With cost data from a previously published [12] micro-costing study of WTW conducted from the service provider and societal perspectives, we undertake an incremental cost-effectiveness analysis to determine the cost per outcome gained for each subsequent arm of the trial.

The literature surrounding cost-effectiveness analyses (CEAs) of prevention and treatment for cocaine and alcohol abuse continues to grow $[13,14,15]$. One article [16] examines the costeffectiveness of a screening and brief intervention for alcohol abuse in a primary care setting. Others $[15,17,18,19]$ address the additional costs of voucher-based and prize-based contingency management programs above and beyond standard treatment for cocaine and opioid dependence. Two CEAs performed analyses alongside RCTs for alcohol treatment, one comparing social 
behavior and network therapy to motivational enhancement therapy [20], and another examining combined behavioral and pharmacotherapies [13]. An additional analysis illustrated the incremental cost-effectiveness of an enhanced, personalized HIV intervention compared to SI for preventing drug use [21].

Decision and policy makers need to be aware of interventions' impact on both cocaine and alcohol abuse because the two substances are often co-abused. Of the 1,648 cocaine-dependent patients in the national Drug Abuse Treatment Outcomes Studies (DATOS), 51\% were also alcohol-dependent [22] and in a study of 158 alcohol-dependent outpatients, 62 of them also manifested cocaine abuse [23]. This study was conducted in accordance with the recommendations of the US Panel on Cost-Effectiveness in Health and Medicine [24].

\section{Methods}

The protocol for this study and supporting CONSORT checklist and flow diagram are available as supporting information; see Checklist S1, Protocol S1, and Flow Diagram S1.

\section{Ethics Statement}

This study has been approved by IRB Human Studies Committee at Washington University Hilltop (03-20), Washington University Medical Center (04-0285) and Yale University Human Investigation Committee (27312). The randomized controlled trial in the parent study has been registered with ClinicalTrials.gov and the registration number is 01235091 . Data from the parent study was analyzed anonymously; written consent was obtained for the effectiveness data and for the cost data.

\section{Recruitment, Design, and Sample}

Community health outreach workers recruited study participants from targeted recruitment zones, employing street-outreach methods based on prior work [25]. Eligibility criteria included: (1) being a woman over the age of 18; (2) reporting sexual activity in the prior four months; (3) using cocaine, heroin, amphetamines, or other injection drugs; and (4) residing in the St. Louis metropolitan area during the study period (2000-2006). Of the 501 women in the study, 420 participants received the standard intervention (SI) and completed baseline and both 4-months and 12-months endpoint assessments. They were randomized to one of three intervention conditions: SI alone $(\mathrm{n}=135)$; WWE $(\mathrm{n}=144)$; or 4ES $(\mathrm{n}=141)$. Study participants were $90 \%$ racial/ethnic minorities. On average, they were 39 years old, had approximately 11 years of education, and worked for about 7 months when interviewed at baseline. The number of arrests ranged from an average of 4.9 for WWE participants to 9.4 for SI.

\section{Intervention Conditions and Costs}

Interventions were administered at two community-based outreach centers shared with the St. Louis City Health Department. A peer facilitator - a female in drug recovery with at least one year's sobriety - conducted the SI, which included: (1) a 20-minute session for HIV pre-test counseling, blood collection, and administration of NIDA SI; [26] and (2) two weeks later, blood test results and HIV post-test counseling. The WWE intervention included an additional breast and pelvic examination with cervical cytological testing (Pap smear) provided by a nurse practitioner, who also obtained a short medical history. 4ES provided the additional opportunity of attending four educational sessions delivered by a peer facilitator paired with a health professional. The interactive sessions (1) were based on the Health Belief Model; (2) were focused on reducing unhealthy behaviors; and (3) employed a holistic approach, emphasizing health and nutrition, stress and coping, substance abuse, and HIV/AIDS.

The intervention costs employed in this analysis are based on a microcosting methodology, results of which are reported elsewhere [12].

\section{Effectiveness Measures}

For cocaine use, the outcome measures include: occasions used (number of days used * episodes per day, past 30 days); episodes per day (past 30 days); cocaine free days (past 30 days); participants with negative urine tests; proportion of participants abstaining (past 30 days); and proportion of participants abstaining (past 4 months). For alcohol consumption, we examined the following outcomes: total drinks (past 7 days); drinks per day of the days participants drank alcohol (past 7 days); proportion of participants avoiding heavy drinking (past 7 days; "heavy drinking" defined as $\geq 4$ drinks per day on the days participants drank alcohol); [6] and proportion of participants abstaining (past 30 days).

We estimated incremental effects by a two-step difference-indifferences approach in order to best compare results across trial arms. First, we calculated the difference in effectiveness from 4 months to baseline $(4 \mathrm{M})$ and 12 months to baseline (12 M) within the same intervention. Second, differences within groups were compared to subsequent interventions to obtain the difference in differences, or incremental effects. In cases where a smaller mean from baseline indicates an intervention's effectiveness, the incremental effects were calculated to reflect the greater relative improvement by converting the change to a positive value.

\section{Incremental Cost-Effectiveness Analyses}

We estimated the incremental cost-effectiveness of WWE compared to SI and 4ES compared to WWE by calculating the ratio of the difference in intervention costs (incremental costs) to the resulting incremental benefit (incremental effects). This calculation yields an incremental cost-effectiveness ratio (ICER), which provides the cost per obtaining an additional unit of outcome. An intervention is dominated if it is more costly and less effective than the alternative. Moreover, an intervention is extended dominated if the alternative is more costly, more effective and provides a lower ICER [13,27]. An incremental cost-effectiveness analysis is appropriate in this study because each subsequent intervention adds significant components.

\section{Sensitivity Analyses}

To examine the robustness of our findings and assess sampling variability, we conducted one- and two-way sensitivity analyses. One-way sensitivity analyses varied the costs and outcomes separately. Incremental costs of WWE were examined at $\$ 100$, $\$ 200, \$ 500$ and $\$ 1,000$ per participant. For $4 \mathrm{ES}$, we evaluated alternative scenarios and their corresponding costs if: building rental/utilities decreased by $50 \%(\$ 549)$ and $25 \%$ (\$777); and, if each session had five people $(\$ 759)$ and three people $(\$ 1,233)$, rather than four [12]. We varied outcomes through statistically significant ranges of $5 \%$ intervals, determining when an intervention would become dominated or would no longer be dominated ("switching point"), depending on the base case. WWE and 4ES were also varied to achieve the following clinically significant levels: 12 and 15 occasions of cocaine use ("moderate to high" use); [28] 1 and 0.5 episodes of cocaine use per day (an approximate range of "moderate" use); 3 weeks of cocaine abstinence; [29] 85\% cocaine free days (15\% days used); [30] and, one and four drink(s) per day on the days participants drank alcohol (a range for "sporadic" and "heavy" drinkers) [31]. In two-way sensitivity analyses we varied these parameters simultaneously. 
Table 1. Effectiveness Measures by Treatment Group.

\begin{tabular}{|c|c|c|c|}
\hline \multirow[b]{3}{*}{ Treatment Outcome } & \multirow{2}{*}{$\frac{\text { SI }}{(n=135)}$} & \multirow{2}{*}{$\begin{array}{l}\text { WWE } \\
(n=144)\end{array}$} & \multirow{2}{*}{$\begin{array}{l}\text { 4ES } \\
(n=141)\end{array}$} \\
\hline & & & \\
\hline & Mean (s.d.) & ) Mean (s.d.) & Mean (s.d.) \\
\hline \multicolumn{4}{|l|}{ Cocaine Use } \\
\hline \multicolumn{4}{|c|}{ Occasions $^{\mathrm{a}}$ Used, past 30 days $^{\mathrm{b}}$} \\
\hline Pre-treatment & $48.91(96.2)$ & $54.51(104.9)$ & $60.84(144.1)$ \\
\hline End of treatment, $4 \mathrm{mo}$ & $19.04(41.2)$ & $24.70(69.6)$ & $24.59(63.3)$ \\
\hline End of treatment, $12 \mathrm{mo}$ & $29.46(62.6)$ & $34.63(71.8)$ & $19.08(38.7)$ \\
\hline \multicolumn{4}{|c|}{ Episodes per Day, past 30 days $^{b}$} \\
\hline Pre-treatment & $3.02(3.8)$ & $3.22(3.8)$ & $3.46(5.7)$ \\
\hline End of treatment, $4 \mathrm{mo}$ & $2.04(3.3)$ & $1.95(3.2)$ & $1.92(3.6)$ \\
\hline End of treatment, $12 \mathrm{mo}$ & $2.29(4.9)$ & $2.56(5.2)$ & $1.91(2.7)$ \\
\hline \multicolumn{4}{|c|}{ Cocaine Free Days, past 30 days } \\
\hline Pre-treatment & $19.21(10.9)$ & $19.33(11.6)$ & $19.66(11.0)$ \\
\hline End of treatment, $4 \mathrm{mo}$ & $25.03(7.4)$ & $24.97(8.3)$ & $24.63(9.1)$ \\
\hline End of treatment, $12 \mathrm{mo}$ & $22.31(9.7)$ & $22.06(10.1)$ & $24.26(7.9)$ \\
\hline \multicolumn{4}{|c|}{ Participants with Negative Urine Tests ${ }^{c}$} \\
\hline Pre-treatment & 21 & 19 & 14 \\
\hline End of treatment, $12 \mathrm{mo}$ & 34 & 32 & 26 \\
\hline \multicolumn{4}{|c|}{ Abstaining, ${ }^{d}$ past 30 days } \\
\hline Pre-treatment & $0.13(0.3)$ & $0.13(0.3)$ & $0.14(0.4)$ \\
\hline End of treatment, $4 \mathrm{mo}$ & $0.43(0.5)$ & $0.44(0.5)$ & $0.50(0.5)$ \\
\hline End of treatment, $12 \mathrm{mo}$ & $0.33(0.5)$ & $0.33(0.5)$ & $0.32(0.5)$ \\
\hline \multicolumn{4}{|c|}{ Abstaining, ${ }^{d}$ past 4 months } \\
\hline Pre-treatment & $0.07(0.3)$ & $0.06(0.2)$ & $0.08(0.3)$ \\
\hline End of treatment, $4 \mathrm{mo}$ & $0.33(0.5)$ & $0.34(0.5)$ & $0.41(0.5)$ \\
\hline End of treatment, $12 \mathrm{mo}$ & $0.28(0.5)$ & $0.31(0.5)$ & $0.30(0.5)$ \\
\hline \multicolumn{4}{|l|}{ Alcohol Consumption } \\
\hline \multicolumn{4}{|c|}{ Total Drinks, past 7 days $^{\mathbf{b}}$} \\
\hline Pre-treatment & $19.26(31.5)$ & $22.02(40.8)$ & $22.41(46.3)$ \\
\hline End of treatment, $4 \mathrm{mo}$ & $11.75(23.0)$ & $15.66(34.6)$ & $12.00(28.5)$ \\
\hline End of treatment, $12 \mathrm{mo}$ & $16.40(27.8)$ & $14.01(24.6)$ & $13.03(23.0)$ \\
\hline \multicolumn{4}{|c|}{ Drinks per Day, ${ }^{e}$ past 7 days $^{b}$} \\
\hline Pre-treatment & $4.55(5.9)$ & $5.07(7.5)$ & $5.38(7.9)$ \\
\hline End of treatment, $4 \mathrm{mo}$ & $3.01(4.8)$ & $4.42(8.2)$ & $3.18(5.2)$ \\
\hline End of treatment, $12 \mathrm{mo}$ & $4.30(7.3)$ & $3.84(5.4)$ & $3.41(4.9)$ \\
\hline \multicolumn{4}{|c|}{ Preventing Heavy Drinkers, ${ }^{\mathrm{f}}$ past 7 days $^{\mathrm{b}}$} \\
\hline Pre-treatment & $0.59(0.49)$ & $0.59(0.49)$ & $0.57(0.50)$ \\
\hline End of treatment, $4 \mathrm{mo}$ & $0.69(0.46)$ & $0.66(0.47)$ & $0.67(0.47)$ \\
\hline End of treatment, $12 \mathrm{mo}$ & $0.60(0.49)$ & $0.63(0.48)$ & $0.62(0.49)$ \\
\hline \multicolumn{4}{|c|}{ Abstaining, ${ }^{9}$ past 30 days $^{\mathrm{b}}$} \\
\hline Pre-treatment & $0.28(0.45)$ & $0.24(0.43)$ & $0.30(0.46)$ \\
\hline End of treatment, $4 \mathrm{mo}$ & $0.41(0.49)$ & $0.41(0.49)$ & $0.41(0.49)$ \\
\hline End of treatment, $12 \mathrm{mo}$ & $0.37(0.49)$ & $0.35(0.48)$ & $0.39(0.49)$ \\
\hline
\end{tabular}

Abbreviations: SI, NIDA's Standard Intervention; WWE, SI plus Well Woman Exam; 4ES, SI, WWE plus four educational sessions.

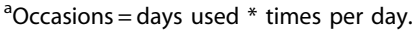

${ }^{\mathrm{b}}$ Missing data from 1-2 patients at some endpoints.

${ }^{\mathrm{C}}$ Measured only at BL and $12 \mathrm{mo}$. The endpoint sample for each intervention included 121, 129 and 129 participants, respectively. Standard deviation not

applicable here because value is number of participants.

${ }^{\mathrm{d}}$ Proportion of participants abstaining from cocaine.

eNumber of drinks per day, of the days participants drank alcohol.
Table 1. Cont.

\author{
Pable 1. Cont. \\ fProportion of participants avoiding heavy drinking. "Heavy drinking" defined \\ as $\geq 4$ drinks per day of the days participants drank alcohol. \\ ${ }^{9}$ Proportion of participants abstaining from alcohol. \\ doi:10.1371/journal.pone.0033594.t001
}

We constructed acceptability curves as an alternative to confidence intervals to address uncertainty in the results $[32,33,34]$. Selecting randomly with replacement ("bootstrapping") from the base case samples yielded 1,000 new samples. From these new outcome means, 1,000 ICERs for each outcome measure were calculated and plotted as a function of willingness to pay (WTP) per additional abstainer. We analyzed the abstinence results because they are the most clinically meaningful outcome. The indicator of cocaine abstainers for the past 30 days provides a better comparison to alcohol abstainers, for which we only have data for the past 30 days. We chose the comparisons between WWE and SI once it became clear they provided better ICERs for both outcomes.

\section{Results}

Incremental Costs and Effects

Table 1 presents treatment outcomes by treatment arm. Table 2 reports the mean differences and percentage change in outcome measures by treatment arm. Each intervention achieved improvements above pre-treatment levels across all outcomes. Tables 3 and 4 provide the incremental effects, indicating the improvements in Table 2 relative to the next least costly intervention. For cocaine abstainers, greater incremental gains were generally achieved at $4 \mathrm{M}$ rather than $12 \mathrm{M}$. For example, both WWE and 4ES achieved incremental improvements at $4 \mathrm{M}$ for cocaine abstainers in the past 30 days and past 4 months, whereas at $12 \mathrm{M}$ only WWE for abstainers in the past 4 months achieved a positive incremental effect. This trend was not universal across all outcomes, however. WWE achieved better outcomes (Table 4) at $12 \mathrm{M}$ than at $4 \mathrm{M}$ for all alcohol outcomes except abstainers. The incremental costs per participant were $\$ 144$ and $\$ 942$ for WWE and 4ES, respectively [12].

\section{Incremental Cost-Effectiveness Analyses: Cocaine Outcomes}

Columns 7-11 of Table 3 report the incremental costeffectiveness ratios across all cocaine outcomes at $4 \mathrm{M}$ and $12 \mathrm{M}$. Comparing WWE to SI at $4 \mathrm{M}$, the cost per additional abstainer (past 30 days) was $\$ 14,445$, but WWE was dominated by SI at $12 \mathrm{M}$. In contrast, at $4 \mathrm{M}$ the ICER for WWE compared to SI of abstainers in the past 4 months was $\$ 7,223$, but only $\$ 3,611$ at $12 \mathrm{M}$. To obtain an additional abstainer (past 4 months) at $4 \mathrm{M}$, WWE compared to SI cost $\$ 1$ 1,624 less than 4ES compared to WWE. Furthermore, an additional abstainer for the past 30 days at $4 \mathrm{M}$ cost $\$ 4,401$ less for WWE compared to SI than for 4ES compared to WWE.

The interventions generated lower ICERs, when not dominated, for additional occasion averted, episode reduced per day, and cocaine free day than for the abstinence outcomes. Moreover, 4ES compared to WWE had lower ICERs for the non-abstinence outcomes at $12 \mathrm{M}$ than at $4 \mathrm{M}$.

\section{Incremental Cost-Effectiveness Analyses: Alcohol Outcomes}

Columns 6-9 of Table 4 report the incremental costeffectiveness ratios across all alcohol outcomes at $4 \mathrm{M}$ and 
Table 2. Mean Differences and Percent Change by Treatment Group at 4 and 12 Months ${ }^{\mathrm{a}}$.

\begin{tabular}{|c|c|c|c|c|c|c|}
\hline \multirow[b]{2}{*}{ Treatment Outcome } & \multicolumn{3}{|l|}{4 months } & \multicolumn{3}{|l|}{12 months } \\
\hline & SI & WWE & 4ES & SI & WWE & 4ES \\
\hline \multicolumn{7}{|l|}{ Cocaine Use } \\
\hline Occasions Used & $-29.87(-61.1 \%)$ & $-29.81(-54.7 \%)$ & $-36.25(-59.6 \%)$ & $-19.45(-39.8 \%)$ & $-19.88(-36.5 \%)$ & $-41.76(-68.6 \%)$ \\
\hline Episodes per Day ${ }^{\mathrm{C}}$ & $-0.98(-32.5 \%)$ & $-1.27(-39.4 \%)$ & $-1.54(-44.5 \%)$ & $-0.73(-24.2 \%)$ & $-0.66(-20.5 \%)$ & $-1.55(-44.8 \%)$ \\
\hline Days Free ${ }^{d}$ & $5.82(30.3 \%)$ & $5.64(29.2 \%)$ & $4.97(25.3 \%)$ & $3.10(16.1 \%)$ & $2.73(14.1 \%)$ & $4.60(23.4 \%)$ \\
\hline Negative Urine Tests ${ }^{\mathrm{e}}$ & $\mathrm{N} / \mathrm{A}$ & $\mathrm{N} / \mathrm{A}$ & N/A & $13.00(61.9 \%)$ & $13.00(68.4 \%)$ & $12.00(85.7 \%)$ \\
\hline Abstaining, past 30 days $^{f}$ & $0.30(231 \%)$ & $0.31(238 \%)$ & $0.36(257 \%)$ & $0.20(154 \%)$ & $0.20(154 \%)$ & $0.18(129 \%)$ \\
\hline Abstaining, past $4 \mathrm{mo}^{\mathrm{g}}$ & $0.26(371 \%)$ & $0.28(467 \%)$ & $0.33(413 \%)$ & $0.21(300 \%)$ & $0.25(417 \%)$ & $0.22(275 \%)$ \\
\hline \multicolumn{7}{|l|}{ Alcohol Consumption } \\
\hline Total Drinks ${ }^{\mathrm{h}}$ & $-7.51(-39 \%)$ & $-6.36(-28.9 \%)$ & $-10.41(-46.5 \%)$ & $-2.86(-14.8 \%)$ & $-8.01(-36.4 \%)$ & $-9.38(-41.9 \%)$ \\
\hline Drinks per Day ${ }^{i}$ & $-1.54(-33.8 \%)$ & $-0.65(-12.8 \%)$ & $-2.20(-40.9 \%)$ & $-0.25(-5.5 \%)$ & $-1.23(-24.3 \%)$ & $-1.97(-36.6 \%)$ \\
\hline $\begin{array}{l}\text { Preventing Heavy } \\
\text { Drinkers }\end{array}$ & $0.10(16.9 \%)$ & $0.07(11.9 \%)$ & $0.10(17.5 \%)$ & $0.01(1.7 \%)$ & $0.04(6.8 \%)$ & $0.05(8.8 \%)$ \\
\hline Abstaining $^{k}$ & $0.13(46.4 \%)$ & $0.17(70.8 \%)$ & $0.11(36.7 \%)$ & $0.09(32.1 \%)$ & $0.11(45.8 \%)$ & $0.09(30.0 \%)$ \\
\hline
\end{tabular}

Abbreviations: SI, NIDA's Standard Intervention; WWE, SI plus Well Woman Exam; 4ES, SI, WWE plus four educational sessions.

${ }^{a}$ Values represent differences and percentage change (parentheses) within groups from baseline. Due to rounding, calculations may not exactly reflect data from Table 1.

${ }^{\mathrm{b}}$ Number of occasions used cocaine (number of days used * times per day); negative number indicates improvement.

'Number of episodes used cocaine per day; negative number indicates improvement.

${ }^{\mathrm{d}}$ Number of days free from cocaine; positive number indicates improvement.

e Number of negative urine tests; positive number indicates improvement. We examined baseline and incremental data, but did not perform an incremental costeffectiveness analysis on this outcome due to missing data.

fProportion of participants abstaining from cocaine, past 30 days; positive number indicates improvement.

${ }^{g}$ Proportion of participants abstaining from cocaine, past 4 months; positive number indicates improvement.

${ }^{\mathrm{h}}$ Total number of alcoholic drinks, past 7 days; negative number indicates improvement.

'Mean number of alcoholic drinks per day, of the days participants drank alcohol, past 7 days; negative number indicates improvement.

'Proportion of participants avoiding heavy drinking, past 7 days; "Heavy drinking" defined as $\geq 4$ drinks per day of the days participants drank alcohol; positive number indicates improvement.

kProportion of participants abstaining from alcohol, past 30 days; positive number indicates improvement.

doi:10.1371/journal.pone.0033594.t002

12 M. For cost per abstainer, WWE was cost-effective compared to SI, with an ICER of $\$ 3,611$ at $4 \mathrm{M}$ and $\$ 7,223$ at $12 \mathrm{M}$, whereas WWE dominated $4 \mathrm{ES}$ at both $4 \mathrm{M}$ and $12 \mathrm{M}$. Conversely, WWE was dominated by SI at $4 \mathrm{M}$ for all other alcohol outcomes (drinks, drinks per day, and heavy drinkers prevented).

At 4 M, 4ES compared to WWE cost $\$ 233$ and $\$ 608$ to avoid an additional drink and to reduce an additional drink per day, respectively. Moreover, an additional heavy drinker was prevented by $4 \mathrm{ES}$ compared to WWE at a cost of $\$ 31,410$.

At $12 \mathrm{M}$, only 4ES compared to WWE for the cost per abstainer was dominated. The cost per heavy drinker prevented for 4ES compared to WWE provided the largest ICER $(\$ 94,230)$. The smallest ICER was the cost per additional drink avoided comparing WWE to SI (\$28), whereas comparing 4ES to WWE on the same outcome yielded an ICER of $\$ 688$. WWE compared to SI cost $\$ 4,815$ to prevent an additional heavy drinker. For the cost per reduced drink per day, ICERs were $\$ 147$ for WWE compared to SI and $\$ 1,273$ for $4 \mathrm{ES}$ compared to WWE.

\section{Sensitivity Analyses}

One-way sensitivity analyses. Varying the costs per participant changed the ICERs proportionally (Table S1). At $4 \mathrm{M}$, for cocaine abstainers (past 4 months), the ICER for WWE compared to SI ranged from $\$ 5,000$ to $\$ 50,000$ (base case ICER: $\$ 7,223)$ at $\$ 100$ to $\$ 1,000$ per participant, whereas comparing $4 \mathrm{ES}$ to WWE at costs that varied from $\$ 549$ to $\$ 1,233$ yielded ICERs from $\$ 10,980$ to $\$ 24,660$ (base case: $\$ 18,846)$. At $4 \mathrm{M}$, for alcohol abstainers, WWE ranged from $\$ 2,500$ to $\$ 25,000$ (base case: $\$ 3,611)$ at $\$ 100$ to $\$ 1,000$ per participant and at $12 \mathrm{M}$ the equivalent cost changes yielded an ICER range of $\$ 5,000$ to $\$ 50,000$ (base case: $\$ 7,223$ ). For WWE at incremental costs of $\$ 500$ or $\$ 1,000$ (base case: $\$ 144.45$ ), excluding dominated options, only cocaine abstainers in the past 30 days at $4 \mathrm{M}$ yielded ICERs above $\$ 50,000$. Generating the smallest ICERs, the cost per cocaine occasion averted for $4 \mathrm{ES}$ at $12 \mathrm{M}$ varied from $\$ 25$ to $\$ 56$.

Varying the effectiveness parameters across statistically (Table 5) and clinically significant ranges (Table 6) indicated the sensitivity of each finding. Some outcomes required greater changes than others to achieve a switching point. For example, 4ES required a $115 \%$ effectiveness decrease at $12 \mathrm{M}$ and a $30 \%$ decrease at $4 \mathrm{M}$ in reducing occasions of cocaine use and a $50 \%$ decrease at $12 \mathrm{M}$ and a $15 \%$ decrease at $4 \mathrm{M}$ in reducing an episode of cocaine use per day to become dominated by WWE. If WWE obtained 10\% fewer cocaine abstainers (past 4 months), it was dominated by SI at $4 \mathrm{M}$ but was still cost-effective at $12 \mathrm{M}$ (\$16,050; Table S2) and did not become dominated by SI until its effectiveness was decreased by $15 \%$. Moreover, 4ES remained cost-effective in reducing a drink per day at $4 \mathrm{M}$ up to the point at which its effectiveness decreased by $50 \%$, while WWE at $4 \mathrm{M}$ required a $25 \%$ increase in effectiveness to eliminate SI domination.

Conversely, an increase or decrease of as low as 5\% revealed a switching point for other cocaine and alcohol outcomes. For example, WWE was dominated by $\mathrm{SI}$ at $4 \mathrm{M}$ and $12 \mathrm{M}$ for cocaine free days in the base case; however, a $5 \%$ increase in 


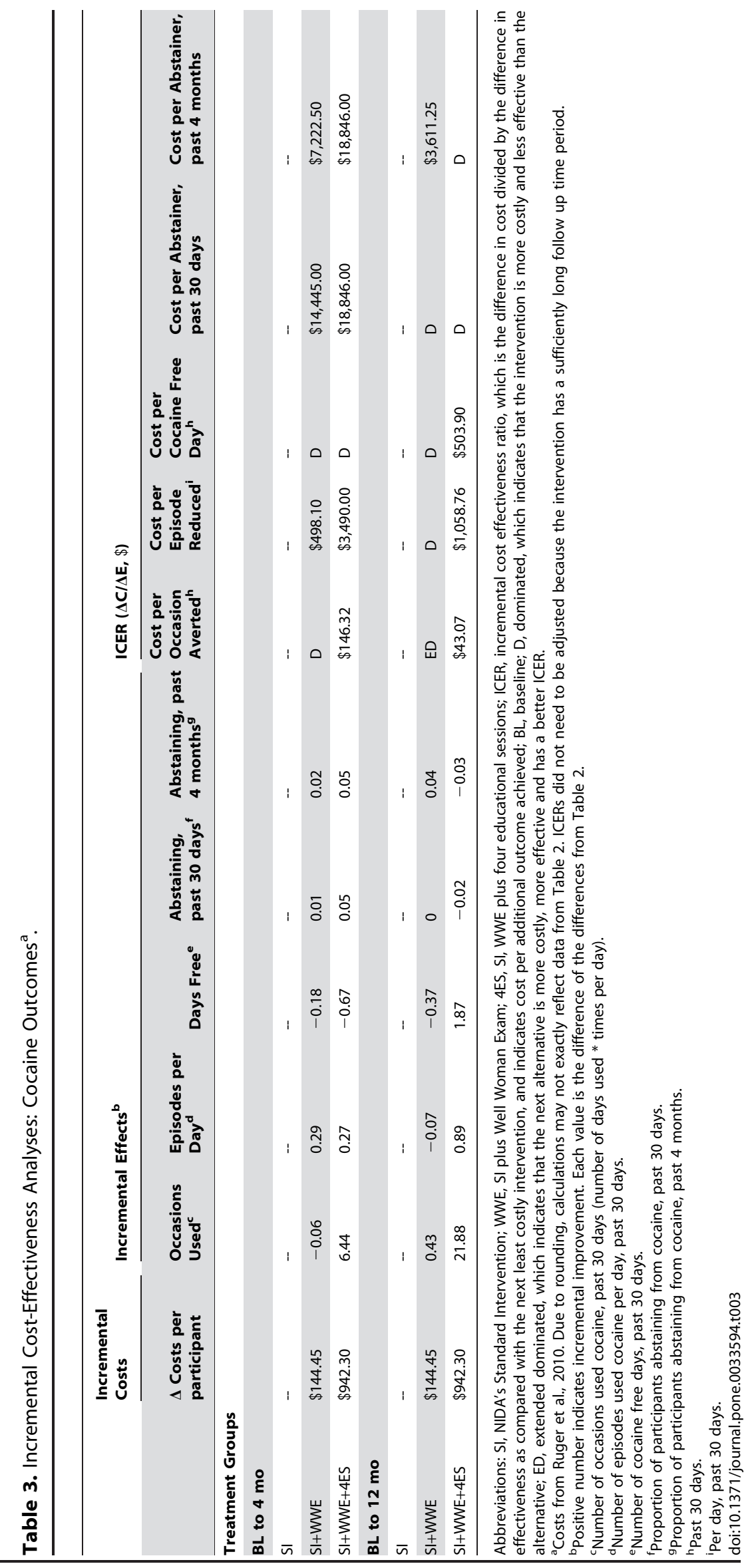




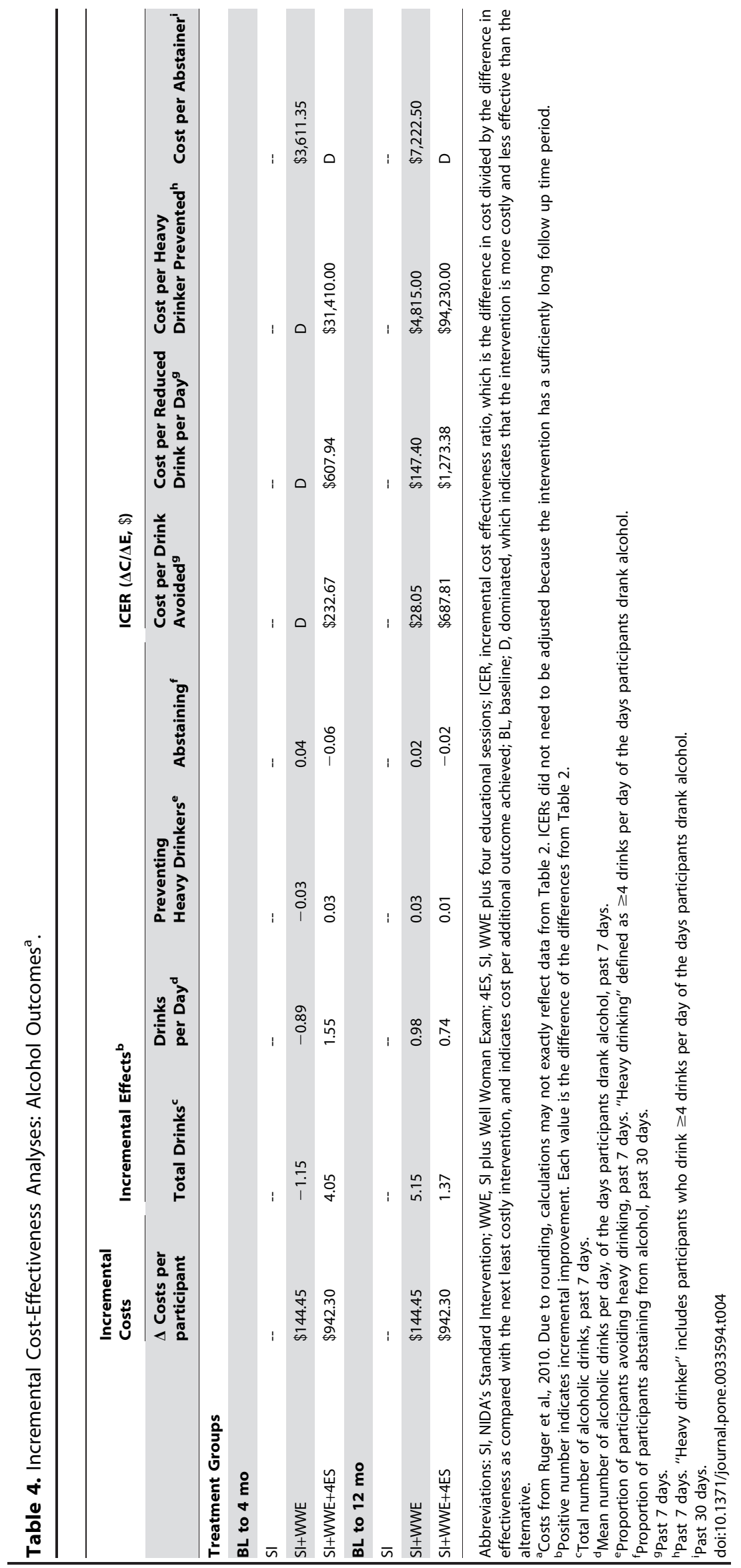


Table 5. One-Way Sensitivity Analyses of Switching Points: Outcomes.

\begin{tabular}{|c|c|c|c|c|}
\hline \multirow[b]{2}{*}{ Parameter Varied $^{\mathbf{a}}$} & \multicolumn{2}{|c|}{ ICER $(\Delta C / \Delta E, \$), 4$ mo } & \multicolumn{2}{|c|}{ ICER $(\Delta C / \Delta E, \$), 12 \mathrm{mo}$} \\
\hline & B-A & C-B & B-A & C-B \\
\hline \multicolumn{5}{|l|}{ Cocaine Use } \\
\hline Occasions $^{\mathrm{b}}$ Used, past 30 days & $+5 \%(\$ 123)$ & $-30 \%$ & $-5 \%$ & $-115 \%$ \\
\hline Episodes per Day, past 30 days & $-15 \%$ & $-15 \%$ & $+5 \%(\$ 2,491)$ & $-50 \%$ \\
\hline Cocaine Free Days, past 30 days & $+5 \%(\$ 135)$ & $+5 \%(\$ 1,678)$ & $+5 \%(\$ 197)$ & $-10 \%$ \\
\hline Abstaining, past 30 days & $-5 \%$ & $-10 \%$ & $+5 \%(\$ 8,755)$ & $+10 \%(\$ 78,525)$ \\
\hline Abstaining, past 4 months & $-10 \%$ & $-15 \%$ & $-15 \%$ & $+15 \%(\$ 62,820)$ \\
\hline \multicolumn{5}{|l|}{ Alcohol Consumption } \\
\hline Total Drinks, past 7 days & $+10 \%(\$ 347)$ & $-35 \%$ & $-40 \%$ & $-15 \%$ \\
\hline Drinks per Day, past 7 days & $+25 \%(\$ 672)$ & $-50 \%$ & $-30 \%$ & $-25 \%$ \\
\hline Preventing Heavy Drinkers, ${ }^{c}$ past 7 days & $+5 \%(\$ 48,150)$ & $-5 \%$ & $-5 \%$ & $-5 \%$ \\
\hline Abstaining, past 30 days & $-10 \%$ & $+15 \%(\$ 628,200)$ & $-10 \%$ & $+10 \%(\$ 49,595)$ \\
\hline
\end{tabular}

Abbreviations: ICER, incremental cost effectiveness ratio, which is the difference in cost divided by the difference in effectiveness as compared with the next least costly intervention and indicates cost per additional outcome achieved; A, SI intervention; B, WWE intervention; C, 4ES intervention.

Table S2 includes complete results of ICERs at $5 \%$ increments en route to the switching points.

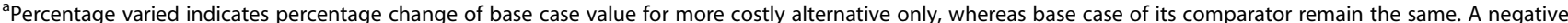
percentage change indicates less effective intervention than the base case, which may or may not indicate a mean lower than the base case (see Table 2). A positive percentage change indicates a more effective intervention than base case. Included in parentheses is the corresponding ICER.

${ }^{\mathrm{b}}$ Occasions $=$ days used ${ }^{*}$ times per day.

c"Heavy drinker" includes participants who drink $\geq 4$ drinks per day of the days participants drank alcohol.

doi:10.1371/journal.pone.0033594.t005

effectiveness for WWE eliminated domination and yielded an ICER of $\$ 135$ at $4 \mathrm{M}$ and $\$ 197$ at $12 \mathrm{M}$.

Two-way sensitivity analyses. We also determined the impact of more than one factor on results (Table S3).

For the cost per additional cocaine abstainer (past 30 days), $4 \mathrm{ES}$ at $4 \mathrm{M}$ yielded ICERs ranging from $\$ 5,490$ to $\$ 12,330$ at incremental costs of $\$ 549$ to $\$ 1,233$ per participant and a $10 \%$ increase in effectiveness. For cocaine free days, a $5 \%$ increase in effectiveness and cost range of $\$ 100$ to $\$ 1,000$ per participant for WWE compared to SI yielded an ICER range of $\$ 94$ to $\$ 936$ at $4 \mathrm{M}$ and $\$ 136$ to $\$ 1,364$ at $12 \mathrm{M}$ (base case: dominated). For alcohol outcomes, relative to SI, WWE would cost $\$ 51,282$ per additional alcohol abstainer at $4 \mathrm{M}$ if the effectiveness of WWE decreased by $5 \%$ and incremental costs increased to $\$ 1,000$ per participant.

Threshold Analyses. Figure 1 illustrates changes in ICERs relative to changes in costs and effectiveness in a stepwise fashion. For cocaine episodes per day, WWE was dominated by SI with a $15 \%$ decrease in effectiveness for WWE, but yielded an ICER under $\$ 2,000$ with an incremental cost of $\$ 100$ and decrease in effectiveness of $10 \%$. At incremental costs of $\$ 500$, WWE was costeffective compared to SI at as large a decrease as $10 \%$ in effectiveness, yielded an ICER under $\$ 2,000$ at base case effectiveness, and under $\$ 500$ if WWE achieved a mean of 1

Table 6. One-Way Sensitivity Analyses of Clinically Significant Outcomes.

\begin{tabular}{|c|c|c|c|c|}
\hline \multirow[b]{2}{*}{ Parameter Varied ${ }^{a}$} & \multicolumn{2}{|c|}{ ICER $(\Delta C / \Delta E, \$), 4 \mathrm{mo}$} & \multicolumn{2}{|c|}{ ICER $(\Delta C / \Delta E, \$), 12 \mathrm{mo}$} \\
\hline & B-A & C-B & B-A & C-B \\
\hline \multicolumn{5}{|l|}{ Cocaine Use } \\
\hline Occasions ${ }^{\mathrm{b}}$ Used, past 30 days -12 & $\$ 11$ & $\$ 50$ & $\$ 6$ & $\$ 33$ \\
\hline Occasions $^{\mathrm{b}}$ Used, past 30 days - 15 & $\$ 15$ & $\$ 59$ & $\$ 7$ & $\$ 36$ \\
\hline Episodes per Day, past 30 days -1 & $\$ 116$ & $\$ 792$ & $\$ 97$ & $\$ 524$ \\
\hline Episodes per Day, past 30 days -0.5 & $\$ 83$ & $\$ 558$ & $\$ 73$ & $\$ 410$ \\
\hline Cocaine Free Days, past 30 days - 3 weeks & $\$ 51$ & $\$ 349$ & $\$ 26$ & $\$ 168$ \\
\hline Cocaine Free Days, past 30 days $-85 \%$ free & $\$ 413$ & $\$ 4,712$ & $\$ 47$ & $\$ 303$ \\
\hline \multicolumn{5}{|l|}{ Alcohol Consumption } \\
\hline Drinks per Day, past 7 days -4 & D & $\$ 1,291$ & $\$ 176$ & $\$ 6,282$ \\
\hline Drinks per Day, past 7 days - 1 & $\$ 57$ & $\$ 253$ & $\$ 38$ & $\$ 299$ \\
\hline
\end{tabular}

Abbreviations: ICER, incremental cost effectiveness ratio, which is the difference in cost divided by the difference in effectiveness as compared with the next least costly intervention and indicates cost per additional outcome achieved; $\mathrm{A}, \mathrm{SI}$ intervention; $\mathrm{B}, \mathrm{WWE}$ intervention; $\mathrm{C}, 4 \mathrm{ES}$ intervention.

Indicates the outcome achieved in the analysis.

${ }^{\mathrm{b}}$ Occasions $=$ days used $*$ times per day.

doi:10.1371/journal.pone.0033594.t006 

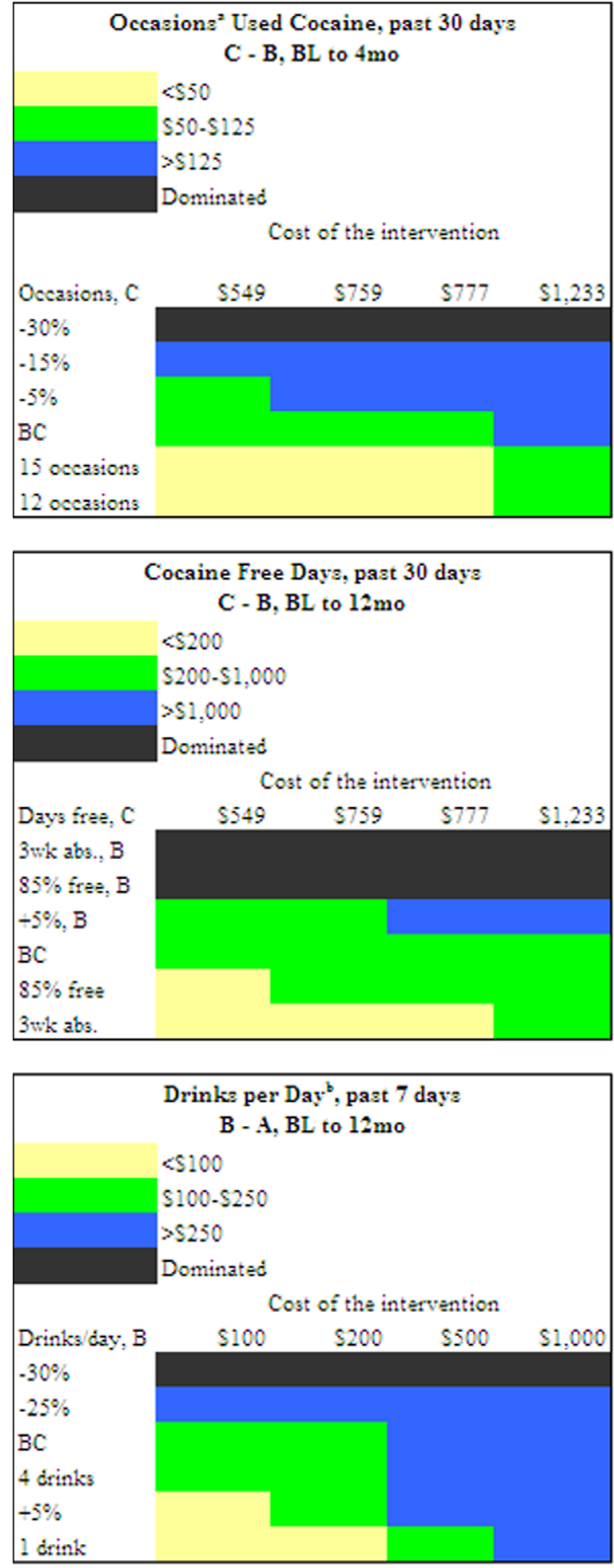
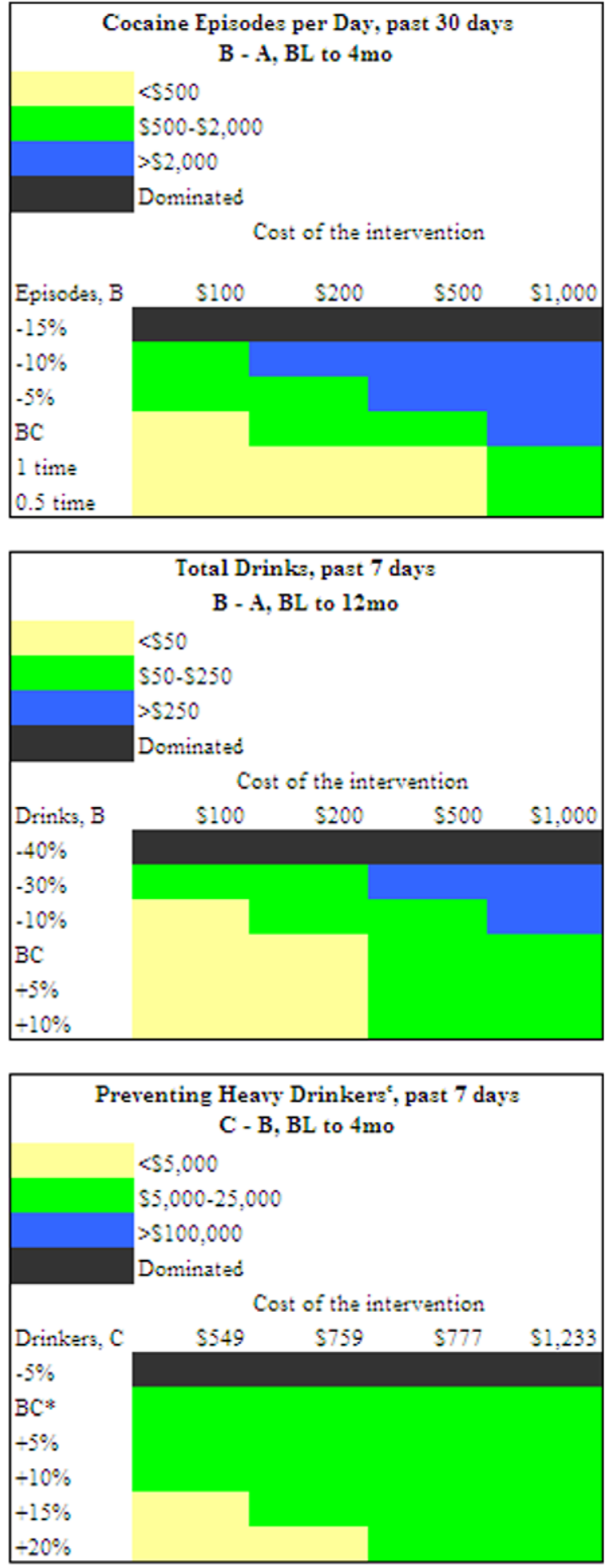

Abbreviations: A, SI intervention; B, SI-WWE intervention; C, SI-WWE 4 ES intervention; BC, base case.

"Oscasions = days used * times per day.

"Drinks per day of the days participants drank alcohol.

"Heavy drinker" includes participants who drink $\geq 4$ drinks per day of the days participants drank alcohol. sensitivity.

Note the gap in the scale to highlight the high sensitivity.

* ICERs for the "Preventing Heavy Drinkers" base case range from $\$ 1 \$, 300$ to $\$ 41,100$, which is over $\$ 25,000$. However, because the range is closer to $\$ 25,000$ than $\$ 100,000$, it is colored the same as the $\$ 5,000-\$ 25,000$ category. 
Figure 1. Two-way Sensitivity Analyses on Cost and Effectiveness. doi:10.1371/journal.pone.0033594.g001

episode per day. 4ES proved highly sensitive for preventing heavy drinkers with an ICER under $\$ 25,000$ across all costs with a 5\% increase in effectiveness.

Acceptability Gurves. Figure $\mathbf{2}$ portrays acceptability curves that address the uncertainty of the results, indicating the probability that WWE is cost-effective when compared to SI at a variety of willingness to pay (WTP) values. For example, the WWE intervention has a 0.5 probability of being cost-effective if society's WTP is $\$ 20,000$ per additional cocaine abstainer (past 30 days) or $\$ 2,600$ per additional alcohol abstainer. If the WTP is $\$ 25,000$, it has a roughly 0.72 probability of being cost-effective for alcohol abstainers. Figure 3 presents willingness to pay depending on intervention costs and changes in intervention effectiveness in achieving per additional cocaine abstainer and alcohol abstainer. For example, a $5 \%$ increase in effectiveness for $4 \mathrm{ES}$ (at a cost of \$549) relative to WWE and for WWE (at a cost of \$500) relative to SI yields a willingness to pay of less than $\$ 10,000$ per additional cocaine abstainer and per additional alcohol abstainer, respectively.

\section{Discussion}

To our knowledge, this is the first cost-effectiveness analysis simultaneously examining cocaine and alcohol abuse in women. The outcome of preventing heavy drinkers proved highly sensitive to changes in effectiveness values. Moreover, the magnitude of an ICER in the base case did not always predict its sensitivity. Decreasing the effectiveness of WWE for alcohol abstainers by $5 \%$ and varying the costs from $\$ 100$ to $\$ 1,000$ yielded significantly different ICER ranges at $4 \mathrm{M}(\$ 5,128-\$ 51,282)$ and at $12 \mathrm{M}$ $(\$ 40,000-\$ 400,000)$, despite comparable base case ICERs $(\$ 3,611$ at $4 \mathrm{M}$ and $\$ 7,223$ at $12 \mathrm{M}$ ). In cases of dominance among cocaine outcomes, the sensitivity to switching points varied considerably. It took only a small improvement $(5 \%)$ in cocaine abstainers (past 30 days) at $12 \mathrm{M}$ for WWE compared to SI to achieve a low ICER $(\$ 8,755)$; yet the same percentage improvement for $4 \mathrm{ES}$ failed to eliminate domination by WWE, and doubling that improvement led to a significantly higher ICER $(\$ 78,525)$. Although SI dominated WWE in the base case for cocaine free days, increasing effectiveness of WWE by $5 \%$ and varying incremental costs from $\$ 100$ to $\$ 1,000$ yielded low ICERs ranging from $\$ 94$ to $\$ 936$ at $4 \mathrm{M}$ and $\$ 136$ to $\$ 1,364$ at $12 \mathrm{M}$.

Our results yielded ICERs that compare favorably to those in the literature, although one needs to be cautious in comparing results across different studies. CEAs alongside randomized controlled trials that examine the cost per obtaining an additional cocaine or alcohol abstainer, aside from analyses of contingency management $(\mathrm{CM})$ programs, are scarce if not non-existent. We identified one study that examined costs and treatment readmission rates [35] and one that looked at costs per abstainer from substance abuse [36], both drawing on calculated ratios of the cost per probability of success. Other studies examined the incremental cost of extending drug abstinence. To extend the longest duration of cocaine and opioid abstinence by an additional week, Olmstead and Petry [15] report costs of $\$ 212$ and $\$ 166$ for voucher-based and prize-based $\mathrm{CM}$ interventions, respectively, compared to standard outpatient treatment. To reduce an additional day of drug use among out-of-treatment substance abusers at risk for HIV, Zarkin et al. [21] measured ICERs ranging from $\$ 36$ to $\$ 140$ for an enhanced intervention compared to SI. Schumacher et al. [37] estimated costs of two addiction interventions compared to usual care to be $\$ 1,007$ and $\$ 1,244$ for an additional week of drug abstinence among homeless persons. For non-abstinence outcomes, Zarkin et al. [13] determined that medical management combined with either naltrexone or naltrexone and acamprosate was cost-effective in avoiding heavy drinking (using the same heavy drinking definition as ours), with ICERs of $\$ 2,847$ and $\$ 8,095$. Jofre-Bonet et al. [38] examined the cost-effectiveness of

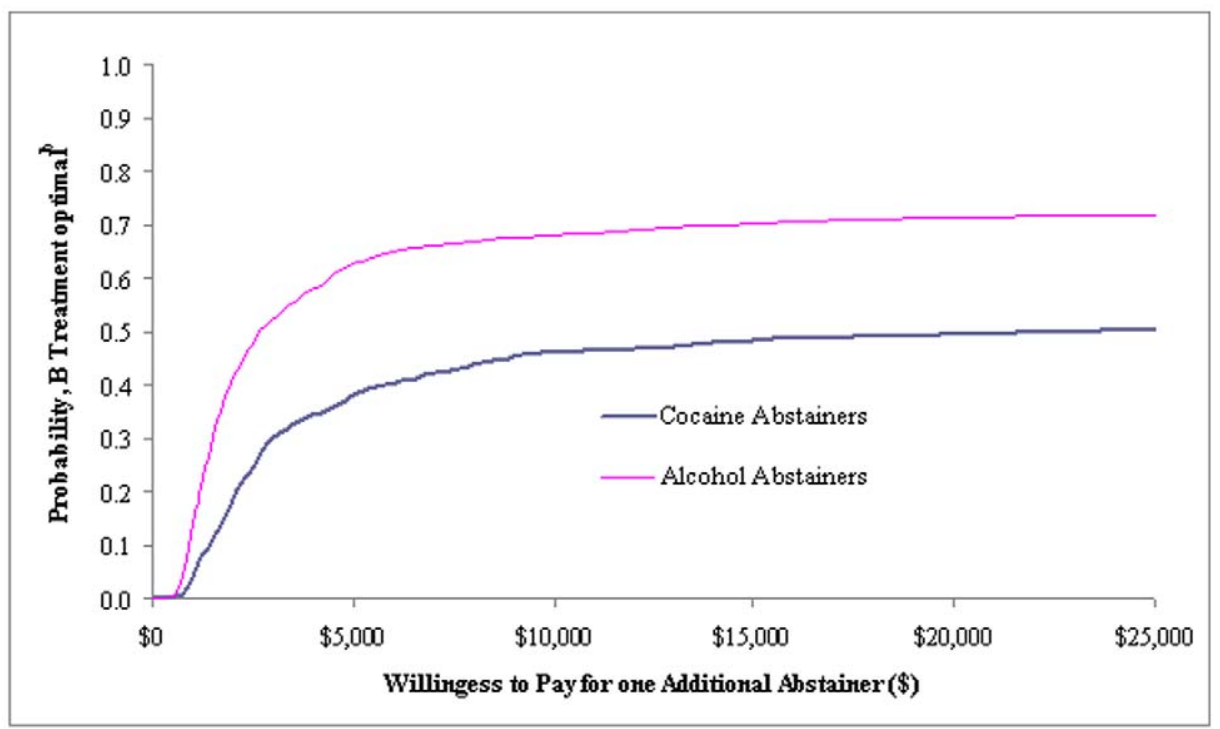

Abbreviations: A, SI intervertion; B, SI+WWE intervertion.

$B-A$, baseline to 4 months, for the past 30 days.

From the 1000 bootstrapped replications, this axis indicates the probability that $B$ is more cost-effective than $A$.

Figure 2. Acceptability Curves.

doi:10.1371/journal.pone.0033594.g002 


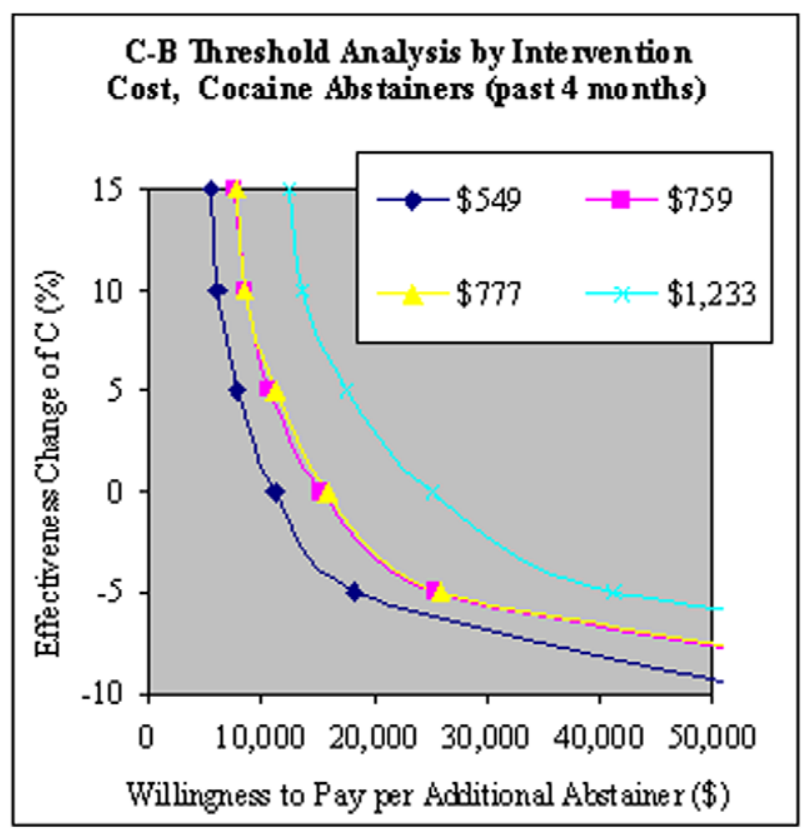

\section{B-A Threshold Analysis by Intervention Cost, Alcohol Abstaine rs}

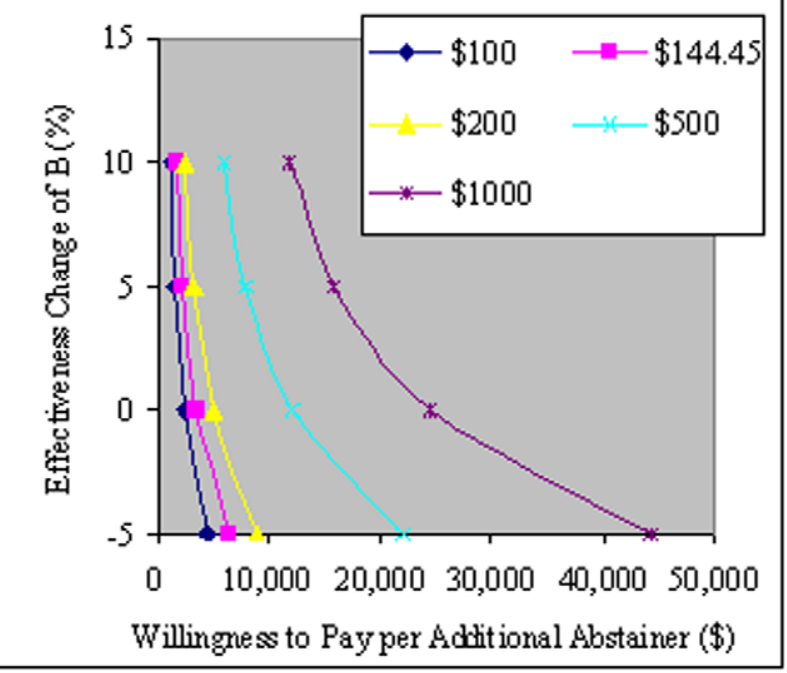

Abbreviations: A, SI intervention; B, SI+WWE intervertion; C, SI+WWE+4ES intervention.

"Incremental vahes calculated from BL to 4 months. Each legend indicats incremental cost of more expersive intervention.

Figure 3. Willingness to Pay per Abstainer.

doi:10.1371/journal.pone.0033594.g003

disulfiram in addition to methadone maintenance for treating cocaine dependence, yielding an incremental cost of $\$ 73$ per day of cocaine reduced.

The cost-effectiveness literature on substance abuse contains comparable analyses for treating heroin abuse, where: 7-day abstinence costs under AUD\$4,000 (US\$2,520) per additional patient for three detoxification methods compared to conventional outpatient treatment, the latter of which was also dominated by buprenorphine; [39] in prison, methadone treatment compared to no treatment costs AUD\$38 (US\$22) per additional heroin free day; [40] buprenorphine was dominated by methadone mainte- nance for obtaining an additional heroin free day [41]. The cost to prevent a recent quitter from smoking (relapse prevented) among low-income pregnant women was $\$ 1,217$ [42].

As a benchmark of comparison for determining the worth of a QALY for alcohol treatment in the UK, two studies [14,20] noted a range consistent with National Institute of Clinical Excellence (NICE) valuation of $£ 20,000-30,000(\$ 37,768-56,652)$ per QALY gained. Additional studies reported the total benefits gained by society, determining the cost-effectiveness of: alcohol prevention [43] and a screening and brief intervention for excessive alcohol use in a primary care setting [16] in terms of QALYs; and 
acamprosate therapy in terms of cost savings per additional abstinent alcoholic [44].

We urge caution, however, not only in comparing our results to QALYs, but also in using QALYs as a measure for substance abuse prevention and treatment in an overarching social utility exercise (e.g. league table) because they overlook broader societal benefits and concerns [45]. As a single quantitative scale, QALYs assign less weight to the disabled and elderly in health care rationing and introduce problems of incommensurability [46,47]. Moreover, since incremental CEAs yield ICERs that are reflective of their comparators, a low ICER may either indicate an ineffective and/or costly comparator or highly effective and/or inexpensive intervention. Thus, it is important, as we did in this study, to compare interventions to clinically relevant comparators. Economic considerations should follow clinical input in the decision making process [46].

The need for further research is clear. In 2003, the U.S. spent $\$ 21$ billion (1.3\% of total health care spending) on treatment for substance use disorders, $\$ 4.83$ billion (23\%) of which was spent by Medicare and Medicaid [48]. By comparison, Medicare costs for end stage renal disease (ESRD), which affected over half a million patients in 2007 [49], were an estimated $\$ 20.8$ billion in 2007 and $\$ 18.1$ billion in 2004 [50] - close to spending on treatment for substance abuse, which affects a much larger population (about 23.1 million in 2008) [1]. ESRD is very expensive to treat, and the relatively small group of ESRD patients deserve medically necessary and appropriate care; in light of the greater population affected by substance abuse and its broad societal costs $(\$ 180.9$ billion for drug abuse in 2002) [4], however, it is sensible to direct additional attention and resources toward identifying and implementing effective and efficient substance abuse treatments. Our study has several limitations. First, generalizability is limited since the study was conducted in St. Louis and targeted drug use and high-risk sexual behaviors in women. Second, substance abuse prevention and treatment positively impact housing and employment sectors [51], costs and frequency of crime [52,53,45], and the development of the children of users [54], suggesting our results underestimated the full economic impact of treatment. Third, the intervention contained components designed for HIV prevention,

\section{References}

1. U.S. Department of Health and Human Services (2009) Results from the 2008 national survey on drug use and health: national findings. NSDUH Series H-36, HHS Publication No. SMA 09-4434. Rockville, MD: Substance Abuse and Mental Health Services Administration, Office of Applied Studies, Division of Population Surveys. Available: http://www.oas.samhsa.gov/nsduh/2k8nsduh/ 2k8Results.pdf. Accessed 2012 February 20.

2. U.S. Department of Health and Human Services (2011) Results from the 2010 national survey on drug use and health: summary of national findings. NSDUH Series H-41, HHS Publication No. (SMA) 11-4658. Rockville, MD: Substance Abuse and Mental Health Services Administration. Available: http://www.oas. samhsa.gov/nsduh/2k10nsduh/2k10Results.pdf. Accessed 2012 February 20.

3. Mokdad AH, Marks JS, Stroup DF, Gerberding.JL (2004) Actual causes of death in the United States, 2000. JAMA 291(10): 1238-1245.

4. Office of National Drug Control Policy (2004) The Economic Costs of Drug Abuse in the United States, 1992-2002. Washington, DC: Executive Office of the President, Available: http://www.ncjrs.gov/ondcppubs/publications/pdf/ economic_costs.pdf. Accessed 2012 February 20.

5. Rawson RA, Huber A, McCann M, Shoptaw S, Farabee D, et al. (2002) A comparison of contingency management and cognitive-behavioral approaches during methadone maintenance treatment for cocaine dependence. Arch Gen Psychiatry 59(9): 817-824.

6. Penberthy JK, Ait-Daoud N, Vaughan M, Fanning T (2010) Review of treatment for cocaine dependence. Curr Drug Abuse Rev 3(1): 49-62.

7. Anton RF, O'Malley SS, Ciraulo DA, Cisler RA, Couper D, et al. (2006) Combined pharmacotherapies and behavioral interventions for alcohol dependence: the COMBINE study: a randomized controlled trial. JAMA 295(17): 2003-2017. rather than exclusively focusing on substance abuse, which reflects important interactions but may not be as targeted as needed for policy and treatment purposes. Fourth, the analysis did not include the incremental differences between 4 months and 12 months, though that information is derivable from data presented. Fifth, our study did not measure the longest duration of abstinence for either cocaine or alcohol. Sixth, the effectiveness outcomes were measured by self report, and biochemical verification was not available for the relevant endpoints used in our CEA.

\section{Supporting Information}

\section{Checklist S1 GONSORT Ghecklist. (DOC)}

\section{Protocol S1 Trial Protocol.}

(DOG)

\section{Flow Diagram S1 GONSORT Flow Diagram.} (TIF)

Table S1 One-way Sensitivity Analyses: Costs. (DOC)

Table S2 One-way Sensitivity Analyses: Outcomes. (DOC)

Table S3 Two-way Sensitivity Analyses. (DOC)

\section{Acknowledgments}

The authors thank Susan Bradford (data control coordinator) and Christina Lazar and Nora Ng (research assistants) for their participation in this study.

\section{Author Contributions}

Conceived and designed the experiments: JR LC. Performed the experiments: JR ABA LC. Analyzed the data: ABA JR LC CL. Contributed reagents/materials/analysis tools: JR ABA CL LC. Wrote the paper: JR CL.

8. Cottler LB, Compton WM, Abdallah AB, Cunningham-Williams R, Abram F, et al. (1998) Peer-delivered interventions reduce HIV risk behaviors among outof-treatment drug abusers. Public Health Rep 113(Suppl 1): 31-41.

9. National Institute on Drug Abuse (2008) Epidemiologic trends in drug abuse, vol. 1. Bethesda, MD: Community Epidemiology Work Group, Available: http://ww2.drugabuse.gov/pdf/cewg/CEWGJune08vol\%201_web.pdf. Accessed 2012 February 20.

10. National Institute on Drug Abuse (2011) Epidemiologic trends in drug abuse. Bethesda, MD: Community Epidemiology Working Group, Available: http://ww2. drugabuse.gov/pdf/cewg/CEWGJan2011_508.pdf. Accessed 2012 February 20.

11. Ruger JP, Abdallah AB, Leukens C, Cottler L (2010) Cost-effectiveness of interventions to prevent HIV and STDs among drug-using women: a randomized controlled trial. Available at SSRN: http://ssrn.com/ abstract $=1619407$ or http://dx.doi.org/10.2139/ssrn.1619407. Accessed 2012 Feb 26.

12. Ruger JP, Abdallah AB, Cottler L (2010) Costs of HIV prevention among outof-treatment drug-using women: results of a randomized controlled trial. Public Health Rep 125(suppl 1): 83-94.

13. Zarkin GA, Bray JW, Aldridge A, Mitra D, Mills MJ, et al. (2008) Cost and costeffectiveness of the COMBINE study in alcohol-dependent patients. Arch Gen Psychiatry 65(10): 1214-1221.

14. Drummond C, Coulton S, James D, Godfrey C, Parrott S, et al. (2009) Effectiveness and cost-effectiveness of a stepped care intervention for alcohol use disorders in primary care: pilot study. Br J Psychiatry 195(5): 448-456.

15. Olmstead TA, Petry NM (2009) The cost-effectiveness of prize-based and voucher-based contingency management in a population of cocaine- or opioiddependent outpatients. Drug Alcohol Depend 102: 108-115. 
16. Tariq L, van den Berg M, Hoogenveen RT, van Baal PHM (2009) Costeffectiveness of an opportunistic screening programme and brief intervention for excessive alcohol use in primary care. PLoS One 4(5): e5696.

17. Olmstead TA, Sindelar JL, Petry NM (2007) Cost-effectiveness of prize-based incentives for stimulant abusers in outpatient psychosocial treatment programs. Drug Alcohol Depend 87(2-3): 175-182.

18. Sindelar J, Elbel B, Petry NM (2007) What do we get for our money? Costeffectiveness of adding contingency management. Addiction 102: 309-316.

19. Sindelar JL, Olmstead TA, Peirce JM (2007) Cost-effectiveness of prize-based contingency management in methadone maintenance treatment programs. Addiction 102: 1463-1471.

20. UKATT Research Team (2005) Cost effectiveness of treatment for alcohol problems: findings of the randomised UK alcohol treatment trial (UKATT). BMJ 331(7516): 544-548.

21. Zarkin GA, Lindrooth RC, Demiralp B, Wechsberg W (2001) The cost and costeffectiveness of an enhanced intervention for people with substance abuse problems at risk for HIV. Health Serv Res 36(2): 335-355.

22. Simpson DD, Joe GW, Fletcher BW, Hubbard RL, Anglin MD (1999) A national evaluation of treatment outcomes for cocaine dependence. Arch Gen Psychiatry 56: 507-514.

23. Echeburua E, De Medina RB, Aizpiri J (2009) Personality disorders among alcohol-dependent patients manifesting or not manifesting cocaine abuse: a comparative pilot study. Subst Use Misuse 44(7): 981-989.

24. Gold MR, Siegel JE, Russell LB, Weinstein MC, eds. Cost-effectiveness in health and medicine. New York: Oxford University Press.

25. Cunningham RM, Cottler LB, Compton WM (1996) Are we reaching and enrolling at-risk drug users for prevention studies? J Drug Issues 26(3): 541-560.

26. Wechsberg WM, MacDonald BR, Dennis ML, Inciardi JA, Surratt HL, et al. (1997) The standard intervention for reduction in HIV risk behavior: protocol changes suggested by the continuing HIV/AIDS epidemic. Bloomington, IL: Chestnut Health Systems/Lighthouse Institute.

27. Resch S, Altice FL, Paltiel AD (2005) Cost-effectiveness of HIV screening for incarcerated pregnant women. J Acquir Immune Defic Syndr 38(2): 163-173.

28. Vocci FJ, Elkashef A (2005) Pharmacotherapy and other treatments for cocaine abuse and dependence. Curr Opin Psychiatry 18(3): 265-270.

29. Dackis CA, Kampman KM, Lynch KG, Pettinati HM, O'Brien CP (2005) A double-blind, placebo-controlled trial of modafinil for cocaine dependence. Neuropsychopharmacology 30: 205-211.

30. McKay JR, Alterman AI, Cacciola JS, Rutherford MJ, O'Brien CP, et al. (1997) Group counseling versus individualized relapse prevention aftercare following intensive outpatient treatment for cocaine dependence: initial results. J Consult Clin Psychol 65(5): 778-788.

31. Gueorguieva R, Wu R, Pittman B, Cramer J, Rosenheck RA, et al. (2007) New insights into the efficacy of naltrexone based on trajectory-based reanalyses of two negative clinical trials. Biol Psychiatry 61(11): 1290-1295.

32. Fenwick E, O'Brien BJ, Briggs A (2004) Cost-effectiveness acceptability curves facts, fallacies and frequently asked questions. Health Econ 13: 405-415.

33. Fenwick E, Claxton K, Sculpher M (2001) Representing uncertainty: the role of cost-effectiveness acceptability curves. Health Econ 10: 779-787.

34. Lothgren M, Zethraeus N (2000) Definition, interpretation and calculation of cost-effectiveness acceptability curves. Health Economics 9: 623-630.

35. Barnett PG, Swindle RW (1997) Cost-effectiveness of inpatient substance abuse treatment. Health Serv Res 32(5): 615-629.

36. Weisner C, Mertens J, Parthasarathy S, Moore C, Hunkeler EM, et al. (2000) The outcome and cost of alcohol and drug treatment in an HMO: day hospital versus traditional outpatient regimens. Health Serv Res 35(4): 791-812.
37. Schumacher JE, Mennemeyer ST, Milby JB, Wallace D, Nolan K (2002) Costs and effectiveness of substance abuse treatments for homeless persons. J Ment Health Policy Econ 5(1): 33-42.

38. Jofre-Bonet M, Sindelar JL, Petrakis IL, Nich C, Frankforter T, et al. (2004) Cost effectiveness of disulfiram: treating cocaine use in methadone-maintained patients. J Subst Abuse Treat 26(3): 225-232.

39. Shanahan MD, Doran GM, Digiusto E, Bell J, Lintzeris N, et al. (2006) A costeffectiveness analysis of heroin detoxification methods in the Australian National Evaluation of Pharmacotherapies for Opioid Dependence (NEPOD). Addict Behav 31(3): 371-387.

40. Warren E, Viney R, Shearer J, Shanahan M, Wodak A, et al. (2006) Value for money in drug treatment: economic evaluation of prison methadone. Drug Alcohol Depend 84(2): 160-166.

41. Harris AH, Gospodarevskaya E, Ritter AJ (2005) A randomised trial of the cost effectiveness of buprenorphine as an alternative to methadone maintenance treatment for heroin dependence in a primary care setting. Pharmacoeconomics 23(1): 77-91.

42. Ruger JP, Weinstein MC, Hammond SK, Kearney MH, Emmons KM (2008) Cost-effectiveness of motivational interviewing for smoking cessation and relapse prevention among low-income pregnant women: a randomized controlled trial. Value Health 11(2): 191-198.

43. Månsdotter AM, Rydberg MK, Wallin E, Lindholm LA, Andréasson S (2007) A cost-effectiveness analysis of alcohol prevention targeting licensed premises. Eur J Public Health 17(6): 618-623.

44. Schädlich PK, Brecht JG (1998) The cost effectiveness of acamprosate in the treatment of alcoholism in Germany. Economic evaluation of the Prevention of Relapse with Acamprosate in the Management of Alcoholism (PRAMA) Study. Pharmacoeconomics 13(6): 719-730.

45. Sindelar JL, Jofre-Bonet M, French MT, McLellan AT (2004) Cost-effectiveness analysis of addiction treatment: paradoxes of multiple outcomes. Drug Alcohol Depend 73(1): 41-50.

46. Ruger JP (2006) Health, capability, and justice: toward a new paradigm of health ethics, policy and law. Cornell J Law Public Policy 15(2): 101-187.

47. Ruger JP (2009) Health and Social Justice. Oxford, UK: Oxford University Press.

48. Mark TL, Levit KR, Coffey RM, McKusick DR, Harwood HJ, et al. (2007) National Expenditures for Mental Health Services and Substance Abuse Treatment, 1993-2003. SAMHSA Publication No. SMA 07-4227. Rockville, MD: Substance Abuse and Mental Health Services Administration.

49. Renalbusiness.com (2009) Many new ESRD patients haven't seen nephrologist. Available: http://www.renalbusiness.com/news/2009/09/many-new-esrdpatients-haven-t-seen-nephrologist.aspx. Accessed 2012 February 20.

50. United States Renal Data System (2009) USRDS 2009 Annual Data Report: Atlas of Chronic Kidney Disease and End-Stage Renal Disease in the United States, vol. 3. Bethesda, MD: National Institutes of Health, National Institute of Diabetes and Digestive and Kidney Diseases.

51. Milby JB, Schumacher JE, Wallace D, Vuchinich R, Mennemeyer ST, et al. (2010) Effects of sustained abstinence among treated substance-abusing homeless persons on housing and employment. Am J Public Health 100(5): 913-918.

52. Basu A, Paltiel AD, Pollack HA (2008) Social costs of robbery and the costeffectiveness of substance abuse treatment. Health Econ 17(8): 927-946.

53. Sindelar JL, Olmstead TA (2006) Illicit drugs and drug-related crime. In: Jones A, ed. The Elgar Companion to Health Economics. Northampton, MA: Edward Elgar Publishing. pp 83-92.

54. Ackerman JP, Riggins T, Black MM (2010) A review of the effects of prenatal cocaine exposure among school-aged children. Pediatrics 125(3): 554-565. 